\title{
Otoneurological findings prevalent in hereditary ataxias
}

\author{
Alterações otoneurológicas prevalentes nas ataxias hereditárias \\ Bianca Simone Zeigelboim', Hélio A. G. Teive², Geslaine Janaina Barbosa Santos ${ }^{3}$, Maria Izabel Rodrigues \\ Severiano ${ }^{3}$, Vinicius Ribas Fonsecaํ, João Henrique Faryniuk', Jair Mendes Marques ${ }^{1}$
}

\begin{abstract}
Objective: To describe and compare the vestibular findings most evident among the hereditary ataxias, as well as correlate their clinical features with the nervous structures affected in this disease. Methods: Seventy-five patients were evaluated and underwent a case history, otorhinolaryngological and vestibular assessments. Results: Clinically, the patients commonly had symptoms of gait disturbances (67.1\%), dizziness (47.3\%), dysarthria (46\%) and dysphagia (36.8\%). In vestibular testing, alterations were predominantly evident in caloric testing (79\%), testing for saccadic dysmetria (51\%) and rotational chair testing (47\%). The presence of alterations occurred in $87 \%$ of these patients. A majority of the alterations were from central vestibular dysfunction (69.3\%). Conclusion: This underscores the importance of the contribution of topodiagnostic labyrinthine evaluations for neurodegenerative diseases as, in most cases, the initial symptoms are otoneurological; and these evaluations should also be included in the selection of procedures to be performed in clinical and therapeutic monitoring.
\end{abstract}

Keywords: spinocerebellar ataxias; vestibular diseases; chronic disease.

\section{RESUMO}

Objetivo: Descrever e comparar os achados vestibulares mais evidentes entre a ataxia hereditária, bem como correlacionar seus aspectos clínicos com o estudo das estruturas nervosas afetadas nesta doença. Métodos: 75 pacientes foram avaliados e submetidos aos seguintes procedimentos: anamnese, avaliação otorrinolaringológica e vestibular. Resultados: Clinicamente, os pacientes apresentaram sintomas de distúrbios da marcha (67,1\%), tonturas (47,3\%), disartria (46\%) e disfagia (36,8\%). No teste vestibular, as alterações foram predominantemente evidentes no teste calórico (79\%), dismetria sacádicas (51\%) e no teste rotatório (47\%). A presença de alterações ocorreu em $87 \%$ dos pacientes. A maioria das alterações observadas foram da disfunção vestibular central (69,3\%). Conclusão: 0 estudo ressalta a importância da contribuição da avaliação labiríntica no topodiagnóstico para doenças neurodegenerativas, uma vez que, na maioria dos casos, os sintomas iniciais são otoneurológicos, e essas avaliações também devem ser incluídas na seleção de procedimentos a serem realizados no monitoramento clínico e terapêutico.

Palavras-chave: ataxias espinocerebelares; doenças vestibulares; doença crônica.

Hereditary ataxias are a heterogeneous group of neurodegenerative diseases that are characterized by the presence of progressive cerebellar ataxia and have initial clinical manifestations such as deterioration of balance and coordination, as well as ocular disorders ${ }^{1,2,3,4}$. Based on genetic inheritance, ataxias can be divided into: 1) autosomal recessive cerebellar ataxias (ARCAs); 2) autosomal dominant cerebellar ataxias, or dominant spinocerebellar ataxias (SCAs); 3) X-linked hereditary ataxia (related to the X chromosome); and 4) mitochondrial ataxias ${ }^{5}$.
Within the group of ARCAs, we highlight Friedreich's ataxia. It is a rare neurodegenerative disease, progressive in nature, and has autosomal recessive early onset in most cases $^{6,7,8}$.

The mutation responsible for the disease is located on chromosome nine, where there is a GAA trinucleotide expansion in the X25 gene. The affected gene has the function of encoding the mitochondrial protein, frataxin, that is involved in iron metabolism ${ }^{7,8,9}$. The deficit of this protein causes the accumulation of iron within the mitochondria disturbing the mitochondrial respiratory chain and increasing

1 Universidade Tuiuti do Paraná, Departamento de Otoneurologia, Curitiba PR, Brasil;

Universidade Federal do Paraná, Hospital de Clínicas, Serviço de Neurologia, Curitiba, PR, Brasil;

${ }^{3}$ Instituto Federal de Educação, Ciência e Tecnologia do Paraná, Curitiba PR, Brasil.

Correspondence: Bianca Simone Zeigelboim; Departamento de Otoneurologia da Universidade Tuiuti do Paraná; R. Gutenberg, 99 / 90 andar; $80420-030$ Curitiba PR, Brasil; E-mail: bianca cwb@yahoo.com.br

Conflict of interest: There is no conflict of interest to declare.

Received 27 July 2017; Received in final form 15 November 2017; Accepted 06 December 2017. 
oxidative stress $^{6,9,10}$. The first symptoms are usually observed in childhood or the early teen years; however, in some cases the diagnosis can be made before two or after 20 years of age. The main features of the disease are: ataxia (impaired coordination) that initially affects the lower limbs and then the upper, absence of tendon reflexes and weakness in the lower limbs, dysarthria, loss of distal deep sensation, and bilateral Babinski sign. Nerve conduction studies show sensory axonal neuropathy ${ }^{9,11}$. Other features associated with this medical condition are: nystagmus, optic atrophy, hearing loss, atrophy in the hands and distal lower limbs, scoliosis, pes cavus, and hammer toes ${ }^{6,11}$.

Within the group of SCAs, we highlight types 2, 3, 6, 7 and 10 , which are of interest in this study. The most common clinical symptoms in SCAs present as gait and appendage ataxia (dysmetria, diadochokinesia in limbs, intentional tremors), dysarthria, nystagmus, ophthalmoplegia, dysphagia, hearing loss (in some patients), pyramidal signs, lower motor neuron syndrome, cognitive dysfunction, epilepsy, visual disturbances (pigmentary retinopathy), peripheral neuropathy, dementia and movement disorders (including parkinsonism, dystonia, myoclonus and chorea) $)^{1,12}$.

The SCA types 2, 3, 6, and 7 are related to unstable CAG repeat expansions in the respective gene, and are classified as polyglutamine disorders ${ }^{1,12,13,14}$. The SCA10 is caused by a repeat expansion of an ATTCT pentanucleotide, in the intron 9 of the gene located in the chromosome 22q13.3.

Different clinical and neuropathological studies in hereditary ataxias have shown that each specific genetic entity has a constellation of signs and symptoms that are related to the duration of the disease and the size of trinucleotide repeat expansion. Thus, the combination of molecular genetic data and clinical signs provide useful information serving as the basis for the genotypic and phenotypic classification of ataxias $^{15}$.

Hereditary ataxias are part of a list of diseases that, by their manifestations and impairment areas, can lead to vestibular disorders, and the labyrinthine examination is an important tool in the confirmation of vestibular disorders and their relationship with the central nervous system ${ }^{16}$.

The aim of this study was to describe and compare the vestibular findings most evident among the hereditary ataxias, as well as correlate their clinical features with the nervous structures affected in this disease.

\section{METHODS}

The research protocol was approved by the Ethics Committee on Research Involving Human Subjects (registration number CEP no. 832.502/2014) following the Brazil Platform. All examinations were performed after formal consent forms were obtained from all participants.
The study had a retrospective cross-sectional design. Seventy-five patients with ataxia were evaluated, 56 with SCAs (26 females and 30 males) and 19 with ARCAs (eight females and 11 males). Among them, 37 patients had a genetically-proven diagnosis of SCAs, and six of Friedreich's ataxia. The diagnosis of ataxia was made by analysis of clinical and genetic tests using the polymerase chain reac$\operatorname{tion}^{17,18}$. This reaction is based on the fact that the oligonucleotides (primers) hybridize specifically to a DNA template strand enabling the production of multiple copies of specific DNA sequences ${ }^{19}$.

The ages of the SCA patients ranged from 18 to 70 years (mean, $42.7 \pm 12.4$ years). The duration of the disease ranged from one to 18 years (mean, $8.4 \pm 4.1$ years) and the ages of the ARCA patients ranged from six to 63 (mean, $36.7 \pm 14.3$ years). The duration of the disease ranged from three to 38 years (mean, 16.2 \pm 9.2 years), as shown in Tables 1 and 2 .

Included in the survey were patients without otoscopic alterations, and excluded were patients with musculoskeletal changes that prevented the examination.

The patients were subjected to the following procedures:

1) Anamnesis: a questionnaire was given with an emphasis on otoneurological signs and symptoms.

2) Otorhinolaryngological assessment: this was performed to rule out any alteration that could affect the test.

3) Vestibular assessment: evaluation of the vestibular function comprises many labyrinthine function and ocular tests. The first part of our patients' evaluation was clinical and consisted of a systematic search for spontaneous, gaze, and positional nystagmus. The second part consisted of interpretation of the vectoeletronystagmography test result, which is the objective register of the variations in the corneoretinal potentials, captured by sensitive electrodes. The vectoeletronystagmography test comprises calibration of ocular movements, search for spontaneous and gaze nystagmus, the oscillatory tracking test, an optokinetic nystagmus search, and rotatory and caloric tests. We performed the vectoeletronystagmography using three-channel equipment (Berger Eletromedicina, model VN316, made in São Paulo, Brazil), a rotating chair (Ferrante, model COD 14200, made in São Paulo, Brazil), a visual stimulator (Neurograff Eletromedicina, model EV VEC, São Paulo, Brazil), and an air caloric stimulator (Neurograff Eletromedicina, model NGR 05, São Paulo, Brazil).

We compared our results with normal standards, obtained from epidemiological studies in the Brazilian population ${ }^{20,21}$. The criteria used to analyze each test, as well as to distinguish central from peripheral vestibulopathy, are shown in Table 3.

\section{Statistical analysis}

We applied the two-proportion z-test to check the most evident symptoms, check which tests presented the most alterations and compare the results of the vestibular examination (analyzing normal and abnormal results) in SCAs and ARCAs and compare them with each other. 
Table 1. Summary of patient demographics and genetic diagnoses in SCAs.

\begin{tabular}{|c|c|c|c|c|c|c|c|c|}
\hline Patient & Age/sex (years) & SCA type & $\begin{array}{c}\text { Disease duration } \\
\text { (years) }\end{array}$ & $\begin{array}{c}\text { Chromosomal locus of } \\
\text { abnormality }\end{array}$ & $\begin{array}{c}\text { Gene } \\
\text { affected }\end{array}$ & $\begin{array}{c}\text { Mutation } \\
\text { type }\end{array}$ & $\begin{array}{l}\text { Protein } \\
\text { affected }\end{array}$ & SARA \\
\hline 1 & $42 / \mathrm{M}$ & SCA3 & 12 & $14 q 32.1$ & ATXN3 & CAG & Ataxin-3 & 4 \\
\hline 2 & $48 / F$ & SCA3 & 15 & $14 q 32.1$ & ATXN3 & CAG & Ataxin-3 & 10 \\
\hline 3 & $43 / \mathrm{M}$ & SCA3 & 12 & $14 q 32.1$ & ATXN3 & CAG & Ataxin-3 & 4.5 \\
\hline 4 & $41 / \mathrm{M}$ & SCA3 & 8 & $14 q 32.1$ & ATXN3 & CAG & Ataxin-3 & 10.5 \\
\hline 5 & $48 / F$ & SCA3 & 10 & $14 q 32.1$ & ATXN3 & CAG & Ataxin-3 & 10.5 \\
\hline 6 & $53 / \mathrm{M}$ & SCA3 & 13 & $14 q 32.1$ & ATXN3 & CAG & Ataxin-3 & 13 \\
\hline 7 & $50 / F$ & SCA3 & 8 & $14 q 32.1$ & ATXN3 & CAG & Ataxin-3 & 9.5 \\
\hline 8 & $30 / F$ & SCA3 & 9 & $14 q 32.1$ & ATXN3 & CAG & Ataxin-3 & 11 \\
\hline 9 & $42 / \mathrm{M}$ & SCA3 & 10 & $14 q 32.1$ & ATXN3 & CAG & Ataxin-3 & 7.5 \\
\hline 10 & $45 / \mathrm{M}$ & SCA3 & 15 & $14 q 32.1$ & ATXN3 & CAG & Ataxin-3 & 11 \\
\hline 11 & $51 / \mathrm{M}$ & SCA3 & 7 & $14 q 32.1$ & ATXN3 & CAG & Ataxin-3 & 1.5 \\
\hline 12 & $45 / M$ & SCA3 & 3 & $14 q 32.1$ & ATXN3 & CAG & Ataxin-3 & 25.5 \\
\hline 13 & $32 / F$ & SCA3 & 5 & $14 q 32.1$ & ATXN3 & CAG & Ataxin-3 & 10 \\
\hline 14 & $46 / F$ & SCA3 & 11 & $14 q 32.1$ & ATXN3 & CAG & Ataxin-3 & 7 \\
\hline 15 & 49/M & SCA2 & 11 & $12 q 24.1$ & ATXN2 & CAG & Ataxin-2 & 33 \\
\hline 16 & $42 / F$ & SCA2 & 8 & $12 q 24.1$ & ATXN2 & CAG & Ataxin-2 & 21.5 \\
\hline 17 & $54 / F$ & SCA2 & 11 & $12 q 24.1$ & ATXN2 & CAG & Ataxin-2 & 28 \\
\hline 18 & $38 / \mathrm{M}$ & SCA2 & 8 & $12 q 24.1$ & ATXN2 & CAG & Ataxin-2 & 4.0 \\
\hline 19 & $41 / \mathrm{M}$ & SCA2 & 12 & $12 q 24.1$ & ATXN2 & CAG & Ataxin-2 & 4.5 \\
\hline 20 & $36 / \mathrm{M}$ & SCA2 & 3 & $12 q 24.1$ & ATXN2 & CAG & Ataxin-2 & 21 \\
\hline 21 & $18 / \mathrm{M}$ & SCA2 & 2 & $12 q 24.1$ & ATXN2 & CAG & Ataxin-2 & 18 \\
\hline 22 & $44 / F$ & SCA2 & 3 & $12 q 24.1$ & ATXN2 & CAG & Ataxin-2 & 21 \\
\hline 23 & $30 / F$ & SCA2 & 10 & $12 q 24.1$ & ATXN2 & CAG & Ataxin-2 & 4 \\
\hline 24 & $42 / M$ & SCA2 & 12 & $12 q 24.1$ & ATXN2 & CAG & Ataxin-2 & 9.5 \\
\hline 25 & $59 / \mathrm{M}$ & SCA6 & 13 & $19 q 13.1$ & CACNA1A & CAG & CACNA1A & 17.5 \\
\hline 26 & $57 / F$ & SCA6 & 5 & $19 q 13.1$ & CACNA1A & CAG & CACNA1A & 4 \\
\hline 27 & $49 / M$ & SCA7 & 13 & 3p14.1 & ATXN7 & CAG & Ataxin-7 & 35 \\
\hline 28 & $47 / F$ & SCA7 & 10 & 3p14.1 & ATXN7 & CAG & Ataxin-7 & 16 \\
\hline 29 & $52 / F$ & SCA10 & 16 & $22 q 13.3$ & ATXN10 & АTTCT & Ataxin-10 & 7 \\
\hline 30 & $30 / \mathrm{M}$ & SCA10 & 4 & $22 q 13.3$ & ATXN10 & АТTCT & Ataxin-10 & 9 \\
\hline 31 & $37 / F$ & SCA10 & 3 & $22 q 13.3$ & ATXN10 & ATTCT & Ataxin-10 & 7 \\
\hline 32 & $49 / F$ & SCA10 & 6 & $22 q 13.3$ & ATXN10 & ATTCT & Ataxin-10 & 16 \\
\hline 33 & $46 / \mathrm{M}$ & SCA10 & 10 & $22 q 13.3$ & ATXN10 & АTTCT & Ataxin-10 & 13 \\
\hline 34 & $27 / F$ & SCA10 & 3 & $22 q 13.3$ & ATXN10 & АTTCT & Ataxin-10 & 14 \\
\hline 35 & $70 / \mathrm{M}$ & SCA10 & 13 & $22 q 13.3$ & ATXN10 & ATTCT & Ataxin-10 & 4 \\
\hline 36 & $54 / \mathrm{M}$ & SCA10 & 11 & $22 q 13.3$ & ATXN10 & АТTCT & Ataxin-10 & 10 \\
\hline 37 & $56 / F$ & SCA10 & 12 & $22 q 13.3$ & ATXN10 & АTTCT & Ataxin-10 & 10 \\
\hline 38 & $63 / F$ & Und. & 10 & - & - & - & - & 7 \\
\hline 39 & $48 / \mathrm{M}$ & Und: & 18 & - & - & - & - & 16 \\
\hline 40 & $58 / F$ & Und. & 10 & - & - & - & - & 4.5 \\
\hline 41 & $35 / F$ & Und. & 5 & - & - & - & - & 9 \\
\hline 42 & $45 / F$ & Und. & 9 & - & - & - & - & 9 \\
\hline 43 & $24 / M$ & Und. & 2 & - & - & - & - & 21 \\
\hline 44 & $27 / M$ & Und. & 7 & - & - & - & - & 7 \\
\hline 45 & $20 / \mathrm{M}$ & Und. & 1 & - & - & - & - & 9.5 \\
\hline 46 & $32 / \mathrm{M}$ & Und. & 5 & - & - & - & - & 18.5 \\
\hline 47 & $22 / \mathrm{M}$ & Und. & 8 & - & - & - & - & 9.5 \\
\hline 48 & $22 / \mathrm{M}$ & Und. & 7 & - & - & - & - & 16 \\
\hline 49 & $62 / \mathrm{M}$ & Und. & 3 & - & - & - & - & 8 \\
\hline 50 & $66 / M$ & Und. & 12 & - & - & - & - & 9.5 \\
\hline 51 & $18 / F$ & Und. & 4 & - & - & - & - & 16 \\
\hline 52 & $23 / \mathrm{M}$ & Und. & 1 & - & - & - & - & 10.5 \\
\hline 53 & $37 / F$ & Und. & 9 & - & - & - & - & 10.5 \\
\hline 54 & $48 / F$ & Und. & 8 & - & - & - & - & 11 \\
\hline 55 & $48 / F$ & Und. & 8 & - & - & - & - & 25 \\
\hline 56 & $51 / F$ & Und. & 7 & - & - & - & - & 25.5 \\
\hline
\end{tabular}


Table 2. Summary of patient demographics and genetic diagnoses in ARCAs.

\begin{tabular}{|c|c|c|c|c|c|c|c|c|}
\hline Patient & $\begin{array}{l}\text { Age/sex } \\
\text { (years) }\end{array}$ & ARCA type & $\begin{array}{c}\text { Disease duration } \\
\text { (years) }\end{array}$ & $\begin{array}{l}\text { Chromosomal locus } \\
\text { of abnormality }\end{array}$ & $\begin{array}{c}\text { Gene } \\
\text { affected }\end{array}$ & $\begin{array}{l}\text { Mutation } \\
\text { type }\end{array}$ & $\begin{array}{l}\text { Protein } \\
\text { affected }\end{array}$ & SARA \\
\hline 1 & $43 / \mathrm{M}$ & FA & 25 & $9 q 13-q 21.1$ & $\times 25$ & GAA & Frataxin & 21 \\
\hline 2 & $41 / \mathrm{M}$ & FA & 7 & $9 q 13-q 21.1$ & $\times 25$ & GAA & Frataxin & 10 \\
\hline 3 & $30 / F$ & FA & 18 & $9 q 13-q 21.1$ & $\times 25$ & GAA & Frataxin & 8 \\
\hline 4 & $24 / M$ & FA & 8 & $9 q 13-q 21.1$ & $\times 25$ & GAA & Frataxin & 10.5 \\
\hline 5 & $29 / M$ & FA & 13 & $9 q 13-q 21.1$ & $\times 25$ & GAA & Frataxin & 14 \\
\hline 6 & $17 / \mathrm{M}$ & FA & 3 & $9 q 13-q 21.1$ & $\times 25$ & GAA & Frataxin & 13 \\
\hline 7 & $63 / F$ & Und. & 38 & - & - & - & - & 7 \\
\hline 8 & $06 / F$ & Und. & 6 & - & - & - & - & 19 \\
\hline 9 & $37 / F$ & Und. & 19 & - & - & - & - & 28 \\
\hline 10 & $41 / F$ & Und. & 20 & - & - & - & - & 11 \\
\hline 11 & $27 / F$ & Und. & 12 & - & - & - & - & 1.5 \\
\hline 12 & $25 / F$ & Und. & 12 & - & - & - & - & 25.5 \\
\hline 13 & $55 / F$ & Und. & 30 & - & - & - & - & 7 \\
\hline 14 & $44 / \mathrm{M}$ & Und. & 10 & - & - & - & - & 3.5 \\
\hline 15 & $55 / M$ & Und. & 12 & - & - & - & - & 28 \\
\hline 16 & $37 / M$ & Und. & 17 & - & - & - & - & 4.0 \\
\hline 17 & $51 / \mathrm{M}$ & Und. & 30 & - & - & - & - & 9.5 \\
\hline 18 & $27 / M$ & Und. & 10 & - & - & - & - & 16 \\
\hline 19 & $46 / M$ & Und. & 18 & - & - & - & - & 10 \\
\hline
\end{tabular}

ARCA: autosomal recessive cerebellar ataxia; Und.: undetermined; M: male; F: female; FA: Friedreich's ataxia; SARA: scale for the assessment and rating of ataxia.

Table 3. Normal standards and criteria used to analyze the vestibular tests and distinguish central from peripheral20,21.

\begin{tabular}{|c|c|c|c|}
\hline Variable & Normal Vestibular Exam & Peripheral Vestibular Exam & Central Vestibular Exam \\
\hline $\begin{array}{l}\text { Position nystagmus } \\
\text { (Brandt \& Daroff's } \\
\text { maneuver) }\end{array}$ & Absent & $\begin{array}{l}\text { Present (rotatory, horizontal } \\
\text { rotatory, and oblique) with latency, } \\
\text { paroxysm, weariness, and vertigo }\end{array}$ & $\begin{array}{c}\text { Present (vertical inferior, superior, rotatory, } \\
\text { horizontal rotatory, and oblique), without latency, } \\
\text { paroxysm, weariness, and vertigo }\end{array}$ \\
\hline $\begin{array}{l}\text { Calibration of the } \\
\text { ocular movements }\end{array}$ & Regular & Regular & $\begin{array}{l}\text { Irregular (alterations in latency, accuracy, and } \\
\text { velocity of the saccadic movements) }\end{array}$ \\
\hline $\begin{array}{l}\text { Spontaneous } \\
\text { nystagmus }\end{array}$ & $\begin{array}{l}\text { Present }(<7 \text { degrees } / \text { sec }) \\
\text { with closed eyes; absent } \\
\text { with open eyes. }\end{array}$ & $\begin{array}{c}\text { Present (> } 7 \text { degrees/sec) with } \\
\text { closed eyes; absent with open eyes. }\end{array}$ & $\begin{array}{l}\text { Present with open eyes (vertical inferior, } \\
\text { superior, rotatory, horizontal rotatory, oblique, } \\
\text { cyclic, dissociated, and convergence-retraction) }\end{array}$ \\
\hline Gaze nystagmus & Absent & Absent & $\begin{array}{l}\text { Present, unidirectional, bidirectional, or mixed; } \\
\text { presents a variety of nystagmus types }\end{array}$ \\
\hline Oscillatory track & Types I and II & Type III & $\begin{array}{l}\text { Type IV (pathognomonic); alterations of } \\
\text { morphology and gain }\end{array}$ \\
\hline $\begin{array}{l}\text { Optokinetic } \\
\text { nystagmus }\end{array}$ & $\begin{array}{l}\text { Symmetrical, }<20 \\
\text { degrees } / \mathrm{sec}\end{array}$ & $\begin{array}{l}\text { Asymmetrical, > } 20 \text { degrees/sec, } \\
\text { having superimposed spontaneous } \\
\text { nystagmus with open eyes that } \\
\text { justifies this alteration }\end{array}$ & $\begin{array}{l}\text { Asymmetrical, }>20 \text { degrees/sec, } \\
\text { absent and reduced }\end{array}$ \\
\hline Rotation test & $\begin{array}{l}>33 \% \text {, after stimulation } \\
\text { of the lateral and superior } \\
\text { semicircular ducts }\end{array}$ & $\begin{array}{c}>33 \% \text {, after stimulation of the } \\
\text { lateral and superior semicircular } \\
\text { ducts }\end{array}$ & $\begin{array}{l}>33 \% \text {, after stimulation of the lateral and } \\
\text { superior semicircular ducts and absence of } \\
\text { induced oblique nystagmus }\end{array}$ \\
\hline \multirow{5}{*}{ Air caloric test } & $\begin{array}{l}\text { Absolute value: between } \\
2 \text { and } 24 \text { degrees/sec }\end{array}$ & $\begin{array}{l}\text { Absolute value: }<2 \text { degrees } / \mathrm{sec} \\
\text { (hyporeflexia), }\end{array}$ & $\begin{array}{c}\text { Absolute value: }\langle 2 \text { degrees/sec (hyporeflexia), }> \\
24 \text { degrees/sec (hyperreflexia) and areflexia }\end{array}$ \\
\hline & Relative values: & $\begin{array}{c}>24 \text { degrees/sec (hyperreflexia) and } \\
\text { areflexia }\end{array}$ & Relative values: \\
\hline & $\begin{array}{l}\text { Labyrinth preponderance } \\
\qquad 41 \%\end{array}$ & Relative values: & Labyrinth preponderance $>41 \%$ \\
\hline & \multirow[t]{2}{*}{$\begin{array}{l}\text { Nystagmus directional } \\
\text { preponderance }<36 \%\end{array}$} & Labyrinth preponderance $>41 \%$ & $\begin{array}{c}\text { Nystagmus directional preponderance }>36 \% \\
\text { (Jongkees formula). }\end{array}$ \\
\hline & & $\begin{array}{l}\text { Nystagmus directional } \\
\text { preponderance }>36 \% \text { (Jongkees } \\
\text { formula) }\end{array}$ & $\begin{array}{l}\text { Different nystagmus types may be observed: } \\
\text { dissociated, inverted, perverted, and absence of } \\
\text { the fast component of the nystagmus }\end{array}$ \\
\hline $\begin{array}{l}\text { Inhibiting effect of } \\
\text { ocular fixation }\end{array}$ & Present & Present & Absent \\
\hline
\end{tabular}


We used the chi-squared test for SCAs and Fisher's exact test for ARCAs, to compare the results of the vestibular examination (analyzing the normal and altered results with gender as the variable). A value of 0.05 or $5 \%$ was established as the rejection level for the null hypothesis.

\section{RESULTS}

The most frequent complaints in the anamnesis were: gait imbalance (71.9\% for SCAs and $52.6 \%$ for ARCAs), dysarthria (49.1\% for SCAs and $36.8 \%$ for ARCAs), dizziness (43.8\% for SCAs and $57.8 \%$ for ARCAs) and dysphagia (36.8\% for both types of hereditary ataxia). It was found that headache was only mentioned by patients with an ARCA (42.1\%), as shown in Table 4.

In the application of the two-proportion z-test, it was shown that there was no significant difference in the proportions of patients in both hereditary ataxias for the most common symptoms: gait imbalance $(p=0.1253)$, dysarthria $(p=0.3546)$, dizziness $(p=0.2933)$ and dysphagia $(p=1.0000)$. But the same test revealed that gait imbalance occurred in a higher proportion of cases in relation to the other symptoms, showing a statistical significance $(\mathrm{p}=0.0142 *)$.

The frequency of abnormal findings in the vestibular evaluation in both types of hereditary ataxias are shown in Table 5. It can be observed in the total values that bilateral vestibular hyporeflexia and saccadic dysmetria were more prevalent for SCAs at $73.2 \%$ and $62.5 \%$ respectively, while the frequency for ARCAs was $47.3 \%$ and $15.7 \%$ respectively. There was no postrotatory nystagmus in either of the hereditary ataxias. However, multiple semi-spontaneous nystagmus and asymmetrical optokinetic nystagmus were found at $50 \%$ and $29 \%$, respectively, in the last two tests for SCAs, while for the ARCAs there was a frequency of $36.8 \%$ in each of these tests. For ARCAs, spontaneous nystagmus with open eyes and bilateral vestibular hyperreflexia showed an alteration in $21 \%$ of patients.

The application of the two-proportion z-test revealed a significant difference between the proportions of patients with bilateral vestibular hyporeflexia and absence of post-rotatory nystagmus for the dominant SCAs total $\left(\mathrm{p}=0.0138^{*}\right)$ and ARCAs total $\left(p=0.0431^{*}\right)$. Comparing the patients in both SCA total and ARCA total groups, the difference was significant only for saccadic dysmetria $(\mathrm{p}=0.0009 \%)$.

Table 4. Symptoms in 75 patients with SCAs and ARCAs.

\begin{tabular}{|c|c|c|c|c|}
\hline \multirow{2}{*}{ Symptoms } & \multicolumn{2}{|c|}{ SCAs } & \multicolumn{2}{|c|}{ ARCAs } \\
\hline & No. patients & Frequency (\%) & No. patients & Frequency (\%) \\
\hline Gait imbalance & 41 & 71.9 & 10 & 52.6 \\
\hline Dysarthria & 28 & 49.1 & 7 & 36.8 \\
\hline Dizziness & 25 & 43.8 & 11 & 57.8 \\
\hline Dysphagia & 21 & 36.8 & 7 & 36.8 \\
\hline Dysphonia & 19 & 33.3 & - & - \\
\hline Hearing Loss & 18 & 31.5 & 3 & 15.7 \\
\hline Headaches & 14 & 24.5 & 8 & 42.1 \\
\hline Falling & 14 & 24.5 & 6 & 31.5 \\
\hline Tingling in extremities & 14 & 24.5 & 3 & 15.7 \\
\hline Diplopia & 13 & 22.8 & 6 & 31.5 \\
\hline Tinnitus & 13 & 22.8 & - & - \\
\hline Depression & 12 & 21.0 & 4 & 21.0 \\
\hline Anxiety & 11 & 19.2 & 4 & 21.0 \\
\hline Pain, radiated to shoulder, arm & 10 & 17.5 & 5 & 26.3 \\
\hline Double vision & 10 & 17.5 & - & - \\
\hline Tremors & 9 & 15.7 & 6 & 31.5 \\
\hline Pain, difficulty in neck movement & 9 & 15.7 & 4 & 21.0 \\
\hline Insomnia & 8 & 14.0 & 1 & 5.2 \\
\hline Fatigue & 8 & 14.0 & 5 & 26.3 \\
\hline Migraine & 5 & 8.7 & - & - \\
\hline Taste alteration & - & - & 1 & 5.2 \\
\hline Olfactory alteration & - & - & 1 & 5.2 \\
\hline Cracking neck & - & - & 3 & 15.7 \\
\hline
\end{tabular}

SCAs: dominant spinocerebellar ataxias; ARCAs: autosomal recessive cerebellar ataxias; $n$ : patients; \%: frequency 
The frequency of the vestibular test results in both hereditary ataxias, highlights the central vestibular dysfunction in all ataxias, as shown in Table 6.

The application of the two-proportion z-test showed a significant difference between the proportions of altered and normal results in SCAs total $\left(\mathrm{p}=0.0000^{*}\right)$ and ARCAs total $(\mathrm{p}=0.0000 *)$.

Regarding gender, the vestibular examination for SCAs showed alterations in males in $90 \%$ of cases and in females in
$81 \%$ of cases. In the ARCAs, this alteration occurred in $83.4 \%$ of males and $100 \%$ of females, as indicated in Table 7.

The application of the chi-squared test showed that there was no significant relationship between altered and normal examinations in males and females $(p=0.5141)$ for SCAs, and the application of Fisher's exact test showed the same kind of result for ARCAs $(p=0.3860)$. Comparing results of altered and normal exams (both sexes) for SCA and ARCA groups resulted in no statistical significance $(p=0.5723)$.

Table 5. Frequency of abnormal findings in the vestibular evaluation in patients with SCAs and ARCAs.

\begin{tabular}{|c|c|c|c|c|c|c|c|c|c|c|c|c|c|c|c|c|c|c|c|c|}
\hline \multirow{3}{*}{ Altered results } & \multicolumn{14}{|c|}{ SCAs } & \multicolumn{6}{|c|}{ ARCAs } \\
\hline & \multicolumn{2}{|c|}{ SCA 2} & \multicolumn{2}{|c|}{ SCA 3} & \multicolumn{2}{|c|}{ SCA 6} & \multicolumn{2}{|c|}{ SCA 7} & \multicolumn{2}{|c|}{ SCA 10} & \multicolumn{2}{|c|}{ Und. } & \multicolumn{2}{|c|}{ SCAsT } & \multicolumn{2}{|c|}{ FA } & \multicolumn{2}{|c|}{ Und. } & \multicolumn{2}{|c|}{ ARCAT } \\
\hline & $n$ & $\%$ & $\mathrm{n}$ & $\%$ & $\mathrm{n}$ & $\%$ & $n$ & $\%$ & $\mathrm{n}$ & $\%$ & $\mathrm{n}$ & $\%$ & $\mathrm{n}$ & $\%$ & $n$ & $\%$ & $\mathrm{n}$ & $\%$ & $\mathrm{n}$ & $\%$ \\
\hline $\begin{array}{l}\text { Bilateral labyrinthine } \\
\text { hyporeflexia }\end{array}$ & 5 & 50.0 & 13 & 92.8 & 2 & 100.0 & 2 & 100.0 & 5 & 55.5 & 14 & 73.6 & 41 & 73.2 & 3 & 50.0 & 6 & 46.1 & 9 & 47.3 \\
\hline Saccadic dysmetria & 7 & 70.0 & 9 & 64.2 & 1 & 50.0 & 1 & 50.0 & 6 & 66.6 & 11 & 57.8 & 35 & 62.5 & 2 & 33.4 & 1 & 7.6 & 3 & 15.7 \\
\hline Rotational nystagmus absent & 5 & 50.0 & 9 & 64.2 & 1 & 50.0 & 2 & 100.0 & 2 & 22.2 & 9 & 47.3 & 28 & 50.0 & 3 & 50.0 & 4 & 30.7 & 7 & 36.8 \\
\hline Multiple gaze nystagmus & - & 0.0 & 4 & 28.5 & 1 & 50.0 & 1 & 50.0 & 5 & 55.5 & 5 & 26.3 & 16 & 29.0 & 4 & 67.0 & 3 & 23.0 & 7 & 36.8 \\
\hline $\begin{array}{l}\text { Optokinetic asymmetrical } \\
\text { nystagmus }\end{array}$ & 2 & 20.0 & 8 & 57.1 & - & 0.0 & 2 & 100.0 & 2 & 22.2 & 2 & 10.5 & 16 & 29.0 & 3 & 50.0 & 4 & 30.7 & 7 & 36.8 \\
\hline Bidirectional gaze nystagmus & - & 0.0 & 2 & 14.2 & - & 0.0 & - & 0.0 & 1 & 11.1 & 3 & 15.7 & 6 & 11.0 & - & - & - & - & - & - \\
\hline Positional nystagmus & 1 & 10.0 & - & 0.0 & 1 & 50.0 & - & 0.0 & 1 & 11.1 & 2 & 10.5 & 5 & 9.0 & - & - & - & - & - & - \\
\hline Unidirectional gaze nystagmus & 2 & 20.0 & 1 & 7.1 & 1 & 50.0 & - & 0.0 & - & 0.0 & - & 0.0 & 4 & 7.2 & - & - & - & - & - & - \\
\hline $\begin{array}{l}\text { Unilateral labyrinthine } \\
\text { hyporeflexia }\end{array}$ & 1 & 10.0 & - & 0.0 & - & 0.0 & - & 0.0 & - & 0.0 & 3 & 15.7 & 4 & 7.2 & - & - & - & - & - & - \\
\hline $\begin{array}{l}\text { Spontaneous nystagmus with } \\
\text { open eyes }\end{array}$ & - & 0.0 & 1 & 7.1 & 1 & 50.0 & - & 0.0 & - & 0.0 & 1 & 5.2 & 3 & 5.4 & 2 & 33.4 & 2 & 15.3 & 4 & 21.0 \\
\hline $\begin{array}{l}\text { Unilateral labyrinthine } \\
\text { hyperreflexia }\end{array}$ & - & - & - & - & - & - & - & - & - & - & - & - & - & - & 1 & 16.7 & - & - & 1 & 5.2 \\
\hline $\begin{array}{l}\text { Bilateral labyrinthine } \\
\text { hyperreflexia }\end{array}$ & - & - & - & - & - & - & - & - & - & - & - & - & - & - & - & - & 4 & 30.7 & 4 & 21.0 \\
\hline
\end{tabular}

SCAs: dominant spinocerebellar ataxias; Und: undetermined;SCAsT: dominant spinocerebellar ataxias total; FA: Friedreich's ataxia;ARCAs: autosomal recessive cerebellar ataxias; ARCAT: autosomal recessive cerebellar ataxia total; n: patients; \%: frequency.

Table 6. Frequency of the vestibular exam results in SCAs and ARCAs.

\begin{tabular}{|c|c|c|c|c|c|c|c|c|c|c|c|c|c|c|c|c|c|c|c|c|}
\hline \multirow{3}{*}{ Vestibular exam } & \multicolumn{14}{|c|}{ SCAs } & \multicolumn{6}{|c|}{ ARCAs } \\
\hline & \multicolumn{2}{|c|}{ SCA 2} & \multicolumn{2}{|c|}{ SCA 3} & \multicolumn{2}{|c|}{ SCA 6} & \multicolumn{2}{|c|}{ SCA 7} & \multicolumn{2}{|c|}{ SCA 10} & \multicolumn{2}{|c|}{ Und. } & \multicolumn{2}{|c|}{ SCAsT } & \multicolumn{2}{|c|}{ FA } & \multicolumn{2}{|c|}{ Und. } & \multicolumn{2}{|c|}{ ARCAT } \\
\hline & $n$ & $\%$ & $\mathrm{n}$ & $\%$ & $n$ & $\%$ & $n$ & $\%$ & $n$ & $\%$ & $\mathrm{n}$ & $\%$ & $\mathrm{n}$ & $\%$ & $n$ & $\%$ & $\mathrm{n}$ & $\%$ & $n$ & $\%$ \\
\hline $\begin{array}{l}\text { Central vestibular } \\
\text { dysfunction }\end{array}$ & 6 & 60.0 & 11 & 78.5 & 2 & 100.0 & 2 & 100.0 & 6 & 66.7 & 14 & 73.7 & 41 & 73.2 & 4 & 66.6 & 7 & 53.8 & 11 & 57.9 \\
\hline $\begin{array}{l}\text { Peripheral vestibular } \\
\text { dysfunction }\end{array}$ & 1 & 10.0 & 3 & 21.5 & - & 0.0 & - & 0.0 & - & 0.0 & 3 & 15.8 & 7 & 12.5 & 2 & 33.4 & 4 & 30.8 & 6 & 31.6 \\
\hline $\begin{array}{l}\text { Normal vestibular } \\
\text { exam }\end{array}$ & 3 & 30.0 & - & 0.0 & - & 0.0 & - & 0.0 & $v$ & 33.3 & 2 & 10.5 & 8 & 14.3 & - & - & 2 & 15.4 & 2 & 10.5 \\
\hline Total & 10 & 100.0 & 14 & 100.0 & 2 & 100.0 & 2 & 100.0 & $y$ & 100.0 & 19 & 100.0 & 56 & 100.0 & 6 & 100.0 & 13 & 100.0 & 19 & 100.0 \\
\hline
\end{tabular}

SCAs: dominant spinocerebellar ataxias; Und: undetermined; SCAsT: dominant spinocerebellar ataxias total; ARCAs: autosomal recessive cerebellar ataxias; FA: Friedreich's ataxia; ARCAT: autosomal recessive cerebellar ataxia total; $n$ : patients; \%: frequency

Table 7. Distribution of patients with SCAs and ARCAs according to the results of the vestibular examination and gender.

\begin{tabular}{|c|c|c|c|c|c|c|c|c|c|c|c|c|}
\hline \multirow{3}{*}{ Exam result } & \multicolumn{6}{|c|}{ SCAs } & \multicolumn{6}{|c|}{ ARCAs } \\
\hline & \multicolumn{2}{|c|}{ Male } & \multicolumn{2}{|c|}{ Female } & \multicolumn{2}{|c|}{ Total } & \multicolumn{2}{|c|}{ Male } & \multicolumn{2}{|c|}{ Female } & \multicolumn{2}{|c|}{ Total } \\
\hline & $n$ & $\%$ & $\mathrm{n}$ & $\%$ & $n$ & $\%$ & $\mathrm{n}$ & $\%$ & $\mathrm{n}$ & $\%$ & $\mathrm{n}$ & $\%$ \\
\hline Abnormal & 27 & 90.0 & 21 & 81.0 & 48 & 85.7 & 10 & 83.4 & 7 & 100.0 & 17 & 89.5 \\
\hline Normal & 3 & 10.0 & 5 & 19.0 & 8 & 14.3 & 2 & 16.6 & - & - & 2 & 10.5 \\
\hline Total & 30 & 100.0 & 26 & 100.0 & 56 & 100.0 & 12 & 100.0 & 7 & 100.0 & 19 & 100.0 \\
\hline
\end{tabular}

SCAs: dominant spinocerebellar ataxias; ARCAs: autosomal recessive cerebellar ataxias; $n$ : patients; \%: frequency 


\section{DISCUSSION}

The symptoms most commonly reported by patients in both types of hereditary ataxia in the multiplicity of its clinical forms have been observed by different authors. Among the most common manifestations that may arise during the course of the disease were gait imbalance, dysarthria, dizziness and dysphagia ${ }^{1,12}$. Nacamagoe et al..$^{22}$ reported that vestibular dysfunction in combination with cerebellar atrophy could contribute significantly to the onset of gait instability, which was part of the initial symptomatology of ataxias.

Houssay $^{23}$ observed in SCA patients that the medial longitudinal fasciculus consisted largely of fibers derived from the vestibular nuclei and was involved in reflexes that allowed the eye to adjust to head movements. Information on the position of the head reach the medial longitudinal fasciculus through its connections with the vestibular nuclei. Before that, the author stated that the medial longitudinal fasciculus fibers terminate in the nuclei of oculomotor nerves III, IV and VI, paired with cranial nerves on the same and opposite sides, which explained the appearance of nystagmus in vestibular symptomatology.

In patients with Friedreich's ataxia, Oppenheimer ${ }^{24}$ observed that the dentate nucleus displayed a moderate loss of neurons and the middle and superior cerebellar peduncles showed reductions in size. Furthermore, a loss of Purkinje cells in the cerebellar vermis and upper neurons in the corresponding portion of the inferior olivary nucleus was verified. The dentate nucleus usually had a severe cell loss. The vestibular and auditory systems were also affected by increased gliosis, especially at the level of the medial vestibular, cochlear and superior olivary nuclei. In addition, the author stated that the nuclei of cranial nerves VII, X and XII also showed a reduction in cell numbers, resulting in weakness of the face muscles, dysarthria and dysphagia. The globus pallidus and subthalamic nuclei were also affected; however, the striatum, thalamus and substantia nigra were spared. The cerebellar cortex was usually unchanged.

Regarding the vestibular examination, the alteration with the highest prevalence in both hereditary ataxias was bilateral vestibular hypofunction with $73.2 \%$ in SCAs and $47.3 \%$ in ARCAs. Saccadic dysmetria and a lack of response in the rotational test ( $62.5 \%$ and $50 \%$ ) were the other most common alterations observed for SCAs. For ARCAs, rotary, optokinetic and semi-spontaneous nystagmus were the other most common alterations, present in $36.8 \%$ of patients. It is noteworthy that several alterations were observed in the various tests that make up the inner ear examination in both types of hereditary ataxias; the ones mentioned above were those that were most prevalent.

Although it is known that oculomotor abnormalities in patients with cerebellar dysfunction exist, it is also known that the cerebellum influences the maintenance of the eccentric portion of the eye, responsible for the smooth pursuit in eye movements, as well as the modulation in amplitude of saccades and visual suppression of caloric nystagmus ${ }^{1}$.

The most common alterations in other studies ${ }^{16,25}$ were the presence of positional nystagmus, irregular eye movement calibration, spontaneous rebound nystagmus, bidirectional and multiple semi-spontaneous nystagmus, abolition of optokinetic nystagmus, pendular tracking type IV, vestibular hyperfunction, absence of inhibitory effect of eye fixation, and Aubry signs in the Barany test. In the damaged neuronal structures, it is known that there is vestibular hypofunction, but little is known about when and why it occurs. A study by Fahey et al. ${ }^{26}$ evaluated 20 patients with Friedreich's ataxia and observed that, despite the normal saccadic speed present, latency was essentially extended. In addition, vestibular alterations were found with a marked reduction in the vestibulo-ocular reflex.

A pathology study ${ }^{27}$ of the vestibular complex and its association with its fiber bundles in four patients with SCAs, revealed that the five-core complex (interstitial, lateral, medial, spinal and superior vestibular nuclei) had suffered neurodegeneration resulting from the disease. This indicated that all bundles of fibers associated with the ascending tract of Deiters inputs, juxtarestiform body, lateral and medial vestibulospinal tracts, medial longitudinal fasciculus, and the vestibular portion of cranial nerve VIII all suffered widespread neuronal loss and atrophy that caused demyelination of the structures. These lesions may explain the brainstem changes, postural instability with imbalance, oculomotor deficits and the presence of the pathological vestibulo-ocular reflex.

Zeigelboim et al. ${ }^{16}$ stated that the loss of hair cells of the ampullary crests and maculae, a decline in the number of nerve cells in the vestibular (or Scarpa's) ganglion, the degeneration of the otoliths, reduced labyrinthine blood flow, progressive depression of neural stability, and the reduction in compensation capacity in the vestibulo-ocular reflex and vestibulospinal reflexes all contribute to the reduction of the speed of the eye tracking motion and rotational and caloric hyporeactivity of the both peripheral and central vestibular systems - characteristics presenting in both hereditary ataxias. Luis et al. ${ }^{28}$ pointed to the vestibulo-ocular reflex alteration as a neurophysiological biomarker for disease severity.

Other studies ${ }^{16,24}$ reported that lesions in the cerebellar vermis caused ataxia of the upper limbs, head tremors, dysmetria, and trembling eye movements. It was this part of the anatomy that showed electrical activity along the length of the eye muscles and neck.

In the present study, when comparing the results of the vestibular examination in both hereditary ataxias, we observed a significant difference in central vestibular dysfunction. These findings agree with other studies ${ }^{25}$ that showed involvement of the central vestibular system, and stress the importance of vestibular evaluations in diseases affecting the posterior fossa. Some authors ${ }^{26}$ stressed that the range of changes in eye movements suggested changes in the 
brain stem, as well as the cortical and vestibular pathways. It is known that the central vestibular pathway comprises the vestibular nuclei in the vestibular area of the brainstem rhomboid fossa, vestibulo-oculomotor, vestibulocerebellar, vestibuloreticular, vestibulocortical, vestibulospinal pathways and their connections with the reticular formation (reflex mechanisms, including autonomous ones) and other areas of the CNS. The CNS quickly organizes and processes visual, vestibular and proprioceptive sensory information in specific areas of the brainstem and cerebellum, which orient head, neck, cervical spine, legs, arms, eyes and all body muscle movements ${ }^{29}$. Regarding gender, there was no literature with which to compare our results.

In conclusion, the most evident neurological symptoms found in this group of patients with hereditary ataxias were gait disturbances, dizziness, dysarthria and dysphagia.

Alterations in vestibular examinations occurred in $87 \%$ of patients, mostly in the caloric test (79\%), with a predominant deficit of central vestibular system dysfunction (70\%).

This underscores the importance of the contribution of topodiagnostic labyrinthine evaluations for neurodegenerative diseases as, in most cases, the initial symptoms are otoneurological.

\section{References}

1. Teive HA, Ashizawa T. Primary and secondary ataxias. Curr Opin Neurol. 2015 Aug;28(4):413-22. https://doi.org/10.1097/WC0.0000000000000227

2. Haerer AF. The neurologic examination. 5th ed. Philadelphia: J.B. Lippincott;1992.

3. Zeigelboim BS, Teive HA, Santos R, Arruda WO, Jurkiewicz AL, Mengelberg H et al. Avaliação audiológica na ataxia espinocerebelar. CoDAS. 2013;25(4):351-7. https://doi.org/10.1590/S2317-17822013005000001

4. Zeigelboim BS, Souza SD, Mengelberg H, Teive HA, Liberalesso PB. Vestibular rehabilitation with virtual reality in spinocerebellar ataxia. Audiol Commun Res. 2013 Apr.-June;18(2):143-7. https://doi.org/10.1590/S2317-64312013000200013

5. Teive HA, Munhoz RP, Arruda WO, Lopes-Cendes I, Raskin S, Werneck LC et al. Spinocerebellar ataxias: genotype-phenotype correlations in 104 Brazilian families. Clinics (Sao Paulo). 2012;67(5):443-9. https://doi.org/10.6061/clinics/2012(05)07

6. Embiruçu EK, Martyn ML, Schlesinger D, Kok F. Autosomal recessive ataxias: 20 types, and counting. Arq Neuropsiquiatr. 2009 Dec;67(4):1143-56. https://doi.org/10.1590/S0004-282X2009000600036

7. Ell J, Prasher D, Rudge P. Neuro-otological abnormalities in Friedreich's ataxia.J Neurol Neurosurg Psychiatry. 1984 Jan;47(1):26-32. https://doi.org/10.1136/jnnp.47.1.26

8. Rinaldi C, Tucci T, Maione S, Giunta A, De Michele G, Filla A. Low-dose idebenone treatment in Friedreich's ataxia with and without cardiac hypertrophy. J Neurol. 2009 Sep;256(9):1434-7. https://doi.org/10.1007/s00415-009-5130-6

9. Albano LM, Zatz M, Kim CA, Bertola D, Sugayama SM,

Marques-Dias MJ et al. Friedreich's ataxia: clinical and molecular study of 25 Brazilian cases. Rev Hosp Clin Fac Med Sao Paulo. 2001 SepOct;56(5):143-8. https://doi.org/10.1590/S0041-87812001000500003

10. Fortuna F, Barboni P, Liguori R, Valentino ML, Savini G, Gellera C et al. Visual system involvement in patients with Friedreich's ataxia. Brain. 2009 Jan;132(Pt 1):116-23. https://doi.org/10.1093/brain/awn269

11. Pandolfo M. Friedreich ataxia. Arch Neurol. 2008 Oct;65(10):1296-303. https://doi.org/10.1001/archneur.65.10.1296

12. Arruda WO. CarvalhoNeto A. Late on set autosomal dominant cerebellar ataxia. Neurobiol. 1991 jan-mar;54(1):35-44.

13. Freud AA. Diagnóstico molecular das ataxias espinocerebelares tipos 1, 2, 3, 6 e 7: estudo populacional e em indivíduos com suspeita clínica [tese]. Curitiba: Universidade Federal do Paraná; 2007.

14. Harada H, Tamaoka A, Watanabe M, Ishikawa K, Shoji S. Downbeat nystagmus in two siblings with spinocerebellar ataxia type 6 (SCA 6). J Neurol Sci. 1998 Oct;160(2):161-3. https://doi.org/10.1016/S0022-510X(98)00250-0

15. Schelhaas HJ, Ippel PF, Beemer FA, Hageman G. Similarities and differences in the phenotype, genotype and pathogenesis of different spinocerebellar ataxias. Eur J Neurol. 2000 May;7(3):309-14. https://doi.org/10.1046/j.1468-1331.2000.00067.x

16. Zeigelboim BS, Jurkiewicz AL, Fukuda Y, Mangabeira-Albernaz PL. [Vestibular disorders in degenerative diseases of the central nervous system structures]. Pro Fono. 2001 Sep;13(2):263-70. Portuguese.

17. Dueñas AM, Goold R, Giunti P. Molecular pathogenesis of spinocerebellar ataxias. Brain. 2006 Jun;129(Pt 6):1357-70. https://doi.org/10.1093/brain/awl081

18. Schöls L, Bauer P, Schmidt T, Schulte T, Riess O. Autosomal dominant cerebellar ataxias: clinical features, genetics, and pathogenesis. Lancet Neurol. 2004 May;3(5):291-304. https://doi.org/10.1016/S1474-4422(04)00737-9

19. Pulst SM. Introduction to medical genetics and methods of DNA testing. In: Pulst SM, editor. Genetics of movement disorders. Amsterdan: Academic Press; 2003. p. 1-18.

20. Mangabeira-Albernaz PL, Ganança MM, Pontes PA. Modelo operacional do aparelho vestibular. In: Mangabeira-Albernaz PL, Ganança MM, editors. Vertigem. 2nd ed. São Paulo: Moderna; 1976. p. 29-36.

21. Ganança CC, Souza JA, Segatin LA, Caovilla HH, Ganança MM. Normal limits of parameters for evaluation with digital electronystagmography neurograff. Acta AWHO. 2000;19:105.

22. Nakamagoe K, Iwamoto Y, Yoshida K. Evidence for brainstem structures participating in oculomotor integration. Science. 2000 May;288(5467):857-9. https://doi.org/10.1126/science.288.5467.857

23. Houssay BA. Fisiologia humana. 7a ed. Buenos Aires: El Ateneo; 1984.

24. Oppenheimer DR. Greenfield's neuropathology. 3rd ed. Chicago: Edward Arnold; 1976.

25. Zeigelboim BS, Teive HA, Carvalho HA, Abdulmassih EM, Marques JM, Cardoso RC. Vestibular findings in autosomal recessive ataxia. Int Tinnitus J. 2013;18(2):156-62. https://doi.org/10.5935/0946-5448.20130020

26. Fahey MC, Cremer PD, Aw ST, Millist L, Todd MJ, White OB et al. Vestibular, saccadic and fixation abnormalities in genetically confirmed Friedreich ataxia. Brain. 2008 Apr;131(4):1035-45. https://doi.org/10.1093/brain/awm323 PMID:18238798

27. Buttner N, Geschwind D, Jen JC, Perlman S, Pulst SM, Baloh RW. Oculomotor phenotypes in autosomal dominant ataxias. Arch Neurol. 1998 Oct;55(10):1353-7. https://doi.org/10.1001/archneur.55.10.1353

28. Luis L, Costa J, Muñoz E, de Carvalho M, Carmona S, Schneider E et al. Vestibulo-ocular reflex dynamics with head-impulses discriminates spinocerebellar ataxias types 1, 2 and 3 and Friedreich ataxia. J Vestib Res. 2016 Jul;26(3):327-34. https://doi.org/10.3233/VES-160579

29. Jurkiewicz AL, Floriani A, Collaço LM, Zeigelboim BS. Anatomia funcional da orelha. In: Zeigelboim BS, Jurkiewicz AL (org). Multidisciplinaridade na otoneurologia. São Paulo: Roca; 2012. p. 19-95. 